\title{
Microsecond long atomistic simulation of supercooled water
}

\author{
Roman Shevchuk and Francesco Rao \\ Freiburg Institute for Advanced Studies, University of Freiburg, Freiburg, Germany.
}

Supercooled water is a metastable phase of liquid water below the melting temperature [1. In this regime, the transition to the solid phase is irreversible once the process is activated. An interesting discussion recently developed on the relationship between crystallization rate and the time scales of equilibration within the liquid phase [2, 3]. Calculations using a coarse grained monatomic model of water, the $\mathrm{mW}$ model, suggested that equilibration of the liquid below the temperature of homogeneous nucleation $T_{H} \approx 225 \mathrm{~K}$ is slower than ice nucleation [3]. This observation has important consequences to a proposed theory of water anomalies, predicting a second critical point below $T_{H}$ where a liquid-liquid phase transition occurs 4. Although it has attracted attention $5]$, this theory is not without problems. If the speed of ice nucleation is faster than liquid relaxation, the liquidliquid transition would loose sense from a thermodynamical point of view, being the liquid phase not equilibrated [2].

Here, a $3 \mu$ s long molecular dynamics simulation of the TIP4P-Ew water model is presented to investigate the relaxation properties of an atomistic model in the supercooled region below $T_{H}$. The length of this calculation is one order of magnitude larger than the $350 \mathrm{~ns}$ used to study freezing with the $\mathrm{mW}$ model $[3]$. A box of 1024 molecules was simulated with GROMACS [9]. The Berendsen barostat [10, velocity rescale thermostat 11] and PME [12] were used for pressure coupling, temperature coupling and long-range electrostatics, respectively. The simulation was run at $190 \mathrm{~K}$ and $1250 \mathrm{~atm}$. These values are close to the estimated liquid-liquid critical point for the TIP4P-Ew [13, congruous with recent calculations on the similar TIP4P/2005 model [6].

In these conditions, freezing was not observed as shown by the timeseries of the potential energy $E_{p}$ (Fig. 1 A). Fluctuations are of the order of $0.5 \mathrm{~kJ} / \mathrm{mol}$ per molecule with no systematic drift. It has been observed that once freezing is activated the energy drifts very quickly to low values of the potential energy, with large energy changes (e.g. roughly 5 and $2 \mathrm{~kJ} / \mathrm{mol}$ per molecule for TIP $4 \mathrm{P}$ at $230 \mathrm{~K}$ [14] and TIP4P/2005 at $242 \mathrm{~K}$ [15], respectively).

The time series of the density $\rho$ and the tetrahedral order parameter $Q_{T}$ [17] are shown in Fig. 1 B B C. They respectively correlate and anticorrelate with the potential energy (Pearson correlation coefficient $r=0.69$ and -0.86). The distributions of both $\rho$ and $Q_{T}$ show an appreciable bump at one of the tails (see right panel of Fig. 1 B-C), suggesting the presence of a subpopulation. For the case of the tetrahedral order parameter, the subpopulation emerges at values around 0.873 (red dashed

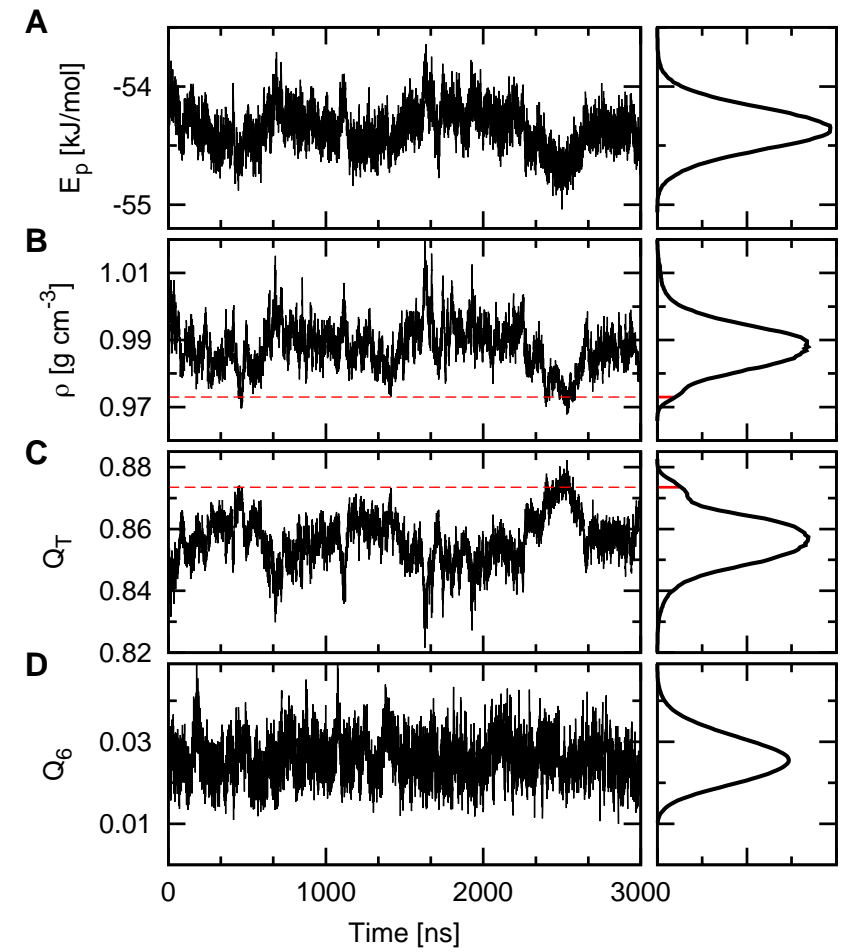

FIG. 1. Time series for the $3 \mu$ s trajectory. (A) potential energy; (B) density; (C) tetrahedral order parameter $Q_{T} ;(\mathrm{D})$ $Q_{6}$ parameter (calculated as in Ref. [2, 16]). Right panels show the probability distribution of the respective quantities. Dashed lines represent deviations from the mean fluctuations, with values of 0.972 and 0.873 for $\rho$ and $Q_{T}$, respectively.

line and right side of Fig. 1 $1 \mathrm{C}$ ). This fluctuation is localized in a time window between 2.3 and $2.6 \mu$ s in correspondence to a decreasing of both the density and the potential energy. It is interesting to note that density subpopulations have been interpreted by some 18 as a signature of the aforementioned liquid-liquid transition.

To check whether this fluctuation corresponded to an ice nucleation attempt, the $Q_{6}$ order parameter 2, 16, 19. was calculated (Fig. 1D). In the time window between 2.3-2.6 $\mu$ s the value of the parameter is around 0.025, with no signs of ice nucleation. Moreover, no correlation with the energy was found $\left(r=10^{-6}\right)$. With a value of $Q_{6}$ for hexagonal ice expected to be one order of magnitude larger [16], no evidence for ice nucleation is found in the present trajectory.

Finally, the oxygen mean-square-displacement (MSD) was calculated (Fig. 2). At timescales shorter than one ns, water shows a subdiffusive behavior (dotted line 




FIG. 2. Oxygen mean square displacement (MSD). The dashed and dotted lines represent a linear and a powerlaw (exponent equal to 0.1 ) regression, respectively. The diffusion coefficient extracted from the linear regime is of $6.6 \times 10^{-9} \mathrm{~cm}^{2} / \mathrm{s}$. The g_msd function of GROMACS was used with 150 windows to improve statistics.

in Fig. 2p. For larger times the system enters a diffusive regime, following the linear relationship MSD $\approx t$ (dashed line), with a maximum average displacement of $3.47 \mathrm{~nm}$ after $3 \mu \mathrm{s}$. Taking into account that the molecular diameter is around $0.3 \mathrm{~nm}$, water molecules have diffused for about 11.5 molecular diameters (the average box side length is of $3.14 \mathrm{~nm}$ ).

In conclusion, evidence is provided that the liquid phase of the TIP4P-Ew model is at equilibrium in the supercooled regime before ice nucleation. Our finding is in agreement with another $\mu$ s long simulation of supercooled water with a 5-site model [18], suggesting that equilibration of the liquid phase below $T_{H}$ is a common feature of atomistic models. The $\mathrm{mW}$ model has shown to reproduce several properties of water, including density and phase diagram [20. But the lack of hydrogens, and consequently of molecular reorientations [21], might considerably speed up the time scales. We speculate that the differences in the relaxation kinetics between atomistic models and the $\mathrm{mW}$ model are due to the lack of molecular reorientations in the latter. Clearly, further ex- perimental validation is needed to clarify which proposed mechanism (if any) is closer to real water.

This work is supported by the Excellence Initiative of the German Federal and State Governments.

[1] O. Mishima and E. H. Stanley, Nature 396, 329 (1998).

[2] D. T. Limmer and D. Chandler, J. Chem. Phys. 135, 134503 (2011).

[3] E. B. Moore and V. Molinero, Nature 479, 506 (2011).

[4] P. H. Poole, F. Sciortino, U. Essmann, and H. E. Stanley, Nature 360, 324 (1992).

[5] Y. Liu, A. Z. Panagiotopoulos, and P. G. Debenedetti, J. Chem. Phys. 131, 104508 (2009).

[6] J. Abascal and C. Vega, J. Chem. Phys. 133, 234502 (2010).

[7] M. Cuthbertson and P. Poole, Phys. Rev. Lett. 106, 115706 (2011).

[8] K. Wikfeldt, C. Huang, A. Nilsson, and L. Pettersson, The Journal of Chemical Physics 134, 214506 (2011).

[9] D. Van Der Spoel, E. Lindahl, B. Hess, G. Groenhof, A. E. Mark, and H. J. C. Berendsen, J. Comput. Chem. 26, 1701 (2005).

[10] H. J. C. Berendsen, J. P. M. Postma, W. F. van Gunsteren, A. DiNola, and J. R. Haak, J. Chem. Phys. 81, 3684 (1984).

[11] G. Bussi, D. Donadio, and M. Parrinello, J. Chem. Phys. 126, 014101 (2007).

[12] T. Darden, D. York, and L. Pedersen, J. Chem. Phys. 98, 10089 (1993).

[13] D. Paschek, A. Rüppert, and A. Geiger, Chem. Eur. J. of Chem. Phys. 9, 2737 (2008).

[14] M. Matsumoto, S. Saito, and I. Ohmine, Nature 416, 409 (2002).

[15] R. Fernández, J. Abascal, and C. Vega, J. Chem. Phys. 124, 144506 (2006).

[16] F. Sciortino, I. Saika-Voivod, and P. Poole, Phys. Chem. Chem. Phys. 13, 19759 (2011).

[17] J. R. Errington and P. G. Debenedetti, Nature 409, 318 (2001).

[18] T. Kesselring, G. Franzese, S. Buldyrev, H. Herrmann, and E. Stanley, Arxiv preprint arXiv:1112.2186 (2011).

[19] P. J. Steinhardt, D. R. Nelson, and M. Ronchetti, Phys. Rev. B 28, 784 (1983).

[20] V. Molinero and E. B. Moore, J. Phys. Chem. B 113, 4008 (2009).

[21] D. Laage and J. Hynes, Science 311, 832 (2006). 